\title{
Numerical simulations of cryogenic jets at supercritical pressure conditions.
}

\author{
M. Jaya Vignesh ${ }^{1}$, Giovanni Tretola ${ }^{1}$, Robert Morgan ${ }^{1}$, Guillaume de Sercey ${ }^{1}$, Konstantina \\ Vogiatzaki ${ }^{1, *}$ \\ ${ }^{1}$ Centre for Automotive Engineering, University of Brighton, United Kingdom \\ ${ }^{*}$ Corresponding author email: k.vogiatzaki@brighton.ac.uk
}

\begin{abstract}
Advancements in novel high efficiency IC engines, cryogenic rocket engines and cooling technologies have given rise to the need for accurate simulation capabilities of cryogenic jets. The ability to accurately simulate cryogenic jets will be pivotal in controlling the jet penetration and/or heat absorption depending upon the role of cryogenic fluids in such systems. In this paper, we present the numerical simulations of injection of two common cryogenic fluids in transportation and energy sector, i.e. $\mathrm{LN}_{2}$ and $\mathrm{CH}_{4}$ injection at supercritical pressures, using the new 'coolFoam' solver developed in-house for cryogenic simulations. The solver is a VoF based compressible two-fluid solver with diffusive mass and heat transfer. Real fluid thermophysical models are utilised to estimate the drastically varying fluid properties across these conditions. This solver enables us to accurately simulate and analyse a range of underlying thermophysical mechanisms in such jets. The different case configurations include working conditions of novel RSCE and cryogenic rocket engines, where fluid is injected as liquid as well as supercritical fluid to understand the pseudoboiling effects. Additionally, multi-specie/fluid configuration corresponding to real world engine chamber conditions, provide a critical insight into the role of thermophysical mechanisms combined with the mixing dynamics.
\end{abstract}

\section{Keywords}

cryogenics, supercritical, real fluid thermophysics, OpenFOAM

\section{Introduction}

Cryogenic fluids find their applications in various technologies such as low emission IC engines (for example in the Recuperated Split Cycle Engine -RSCE- and the Dearman engine), cryogenic rocket engines, cryosurgery, MRI/NMR machines, super-conductors, etc because of their unique themrophysical characteristics. In these technologies, cryogenic fluids are utilised either as coolants (such as $\mathrm{LN}_{2}$ in RSCE [1, 2] and in cryosurgery) or as fuels (such as $\mathrm{LH}_{2}$ and $\mathrm{LCH}_{4}$ in cryogenic rocket engines). Supercritical cryogenic jets in particular are encountered at high pressures in these engineering applications. Although numerical simulations are an integral part in the optimisation of the design of future technologies, existing frameworks are tailored to the simulation of subcritical fluids and not supercritical cryogenic jets. Thus, there is a pressing need for improving the numerical tools that deal with cryogenic and supercritical conditions.

Early cryogenic experiments show the complex nature of cryogenic jets at supercritical conditions where the injected fluid possesses liquid like density, diffuses like a gas and loses the ability to produce droplets and ligaments. This is primarily due to the drastic variation of thermophysical properties around the pseudoboiling point and absence of surface tension. In our previous work we have explained the trends in thermophysical properties of cryogenic fluids at supercritical pressures and provided an overview of the dynamics (mechanics and thermodynamics) of cryogenic jets [2]. Although diffusive transport of mass is usually omitted in numerical simulations of sprays and jets, recent experimental [3] and numerical research [4] have concluded that diffusive transport is important at high pressures, which corresponds to supercritical pressures of cryogenic fluids. In addition, recent theoretical, experimental and 


\begin{tabular}{c|ccc}
\hline & $\begin{array}{c}\text { Pressure } \\
P_{c r}(\mathbf{M P a})\end{array}$ & $\begin{array}{c}\text { Temperature } \\
T_{c r}(\mathrm{~K})\end{array}$ & $\begin{array}{c}\text { Density } \\
\rho_{c r}\left(\mathbf{k g} / \mathbf{m}^{3}\right)\end{array}$ \\
\hline Nitrogen $\left(\mathrm{N}_{2}\right)$ & 3.4 & 126.2 & 313.3 \\
Methane $\left(\mathrm{CH}_{4}\right)$ & 4.6 & 190.6 & 162.7 \\
\hline
\end{tabular}

Table 1. Critical properties of nitrogen and methane

molecular dynamics research has further revealed that supercritical phases cannot be considered as a single homogeneous state and in reality comprises of liquid-like and gas-like phases. Understanding these different supercrtical phases becomes even more complex when multi fluid scenarios are considered. Thus, a numerical framework capable of simulating supercritical cryogenic jets should both a)include a thermophysical model able to approximate the fluid's drastically varying thermophysical properties around pseudo-boiling point and b) be able to simulate the advective as well as diffusive mass and heat transfer.

This paper focuses on investigating the dynamics of single and two fluid supercritical mixtures above and below the pseudo-boiling point upon injection with the use of a novel solver called "CoolFoam" which is designed at the University of Brighton with the necessary capabilities described above. First we introduce a classification of supercritical phases based on the variation of their isobaric thermophysical properties against temperature, which is simple and avoids ambiguities in estimation of the phase boundaries. This classification is utilised to explain important conclusions as it can be seen in the following sections. The simulations are intended to provide deeper insight into characteristics of supercritical cryogenic jets when injected as either cryogenic liquids or as supercritical fluids and the cases represent both single fluid mixing (cryogenic $\mathrm{N}_{2}$ with warm $\mathrm{N}_{2}$ ) and two-fluid mixing (cryogenic $\mathrm{CH}_{4}$ with warmer $\mathrm{N}_{2}$ )

\section{Material and methods Classification of supercritical fluid phases}

At subcritical pressures, liquids vaporise upon reaching the boiling temperature. The phase transition at supercritical pressures is rather different. At supercritical pressures liquids lose their surface tension upon reaching the critical temperature $\left(T_{c r}\right)$ and the fluid beyond this state is classified as "supercritical fluid". A unique feature of supercritical fluids is the continuous transition of thermophysical properties from liquid to gaseous state around the so called pseudoboiling temperature $\left(T_{p b}\right)$ and the heat capacity rise which peaks at $T_{p b}$. This transition is more steep at low supercritical pressures (near $P_{c r}$ ) occurring at a narrow temperature range, while the extent of this transition spreads over a larger temperature range at higher supercritical pressures [5]. The pseudoboiling effect and the variation trend in isobaric thermophysical properties against temperature for various pressures has been explained in our previous publication [2]. The analysis though in this work was restricted to pure fluids and not multi-component mixtures. The thermophysics of mixtures and at mixing conditions is much more complex and less understood.

While the supercritical phase was originally considered a homogeneous state, recent research has identified that supercritical state can be further classified into liquid-like and gas-like supercritical phases. Several boundary lines segregating the supercritical fluids have been proposed including the well known Widom line. In the context of supercritical cryogenic jets, Banuti initially presented a simple classification of the supercritical fluid, based on $T_{p b}$ - liquid-like $\left(T<T_{p b}\right)$, transitional $\left(T \approx T_{p b}\right)$ and gas-like $\left(T>T_{p b}\right)$ [6]. Recently an expanded classification of supercritical fluids based on theoretical, experimental and molecular dynamics simulations is proposed[5]. Since the estimation methods for these phase boundaries are rather scarce, we suggest an alternative classification of the phases at supercritical pressures based on their variation in isobaric thermophysical properties against temperature.

a)The fluid up to the $T_{c r}$ is classified as liquid in accordance with the existing literature whose characteristic feature is the existence of surface tension. b) In order to bound the drastic vari- 
ation in thermophysical properties around $T_{p b}$, we classify the transitional phase as starting from $T_{c r}$ which ends in the temperature where the heat capacity falls back to the value at the $T_{c r}$. c) When the compressibility factor attains a value of 0.95 then the gas-like (supercritical) phase behaves more like an ideal gas and we classify this as ideal gas-like. d)The fluid occupying the region between the transition and ideal gas-like is classified as dense gas-like. Although the thermophysical properties of dense gas-like phase are very close to the ideal gas-like phase, the variation in thermophysical properties against temperature present a rather different trend.

NIST database of thermophysical properties is utilised for classification of supercritical phases of $\mathrm{N}_{2}$ and $\mathrm{CH}_{4}$ (see Fig. 1) at conditions corresponding to the simulation cases.

\section{coolFoam solver}

The coolFoam solver is a novel framework constructed in OpenFOAM based on "one fluid" approach. The solver is designed for compressible non-isothermal two-fluid simulations. The notable characteristics of this solver compared to conventional VoF approaches is the ability to simulate diffusive transport of heat and mass. While conventional VoF approaches consider different phases as separate immiscible fluids and track the primary phase through phase transport equation, a our solver tracks the injected fluid instead, regardless of it's state (or phase) and updates the properties (thermophysical) of the fluid in each cell corresponding to the local state of the fluid (i.e pressure and temperature). The governing equations as will be demonstrated below have been modified in order to remove any requirements of immiscibility. The governing equations solved by the coolFoam solver are volume fraction equation, momentum equation and energy equation. The solver is developed upon a compressible VoF framework, which was presented in our previous publication [2]. The modified volume fraction equation and energy equation utilised by coolFoam to include diffusive mass transfer and the energy exchange due to the diffusive mass transfer are presented below:

- Volume fraction equation:

$$
\frac{\partial \alpha}{\partial t}+\nabla \cdot(\mathbf{U} \alpha)-\nabla \cdot\left(D \nabla \alpha+D_{T} \nabla T\right)=\alpha(1-\alpha)\left(\frac{\psi_{2}}{\rho_{2}}-\frac{\psi_{1}}{\rho_{1}}\right) \frac{D p}{D t}+\alpha \nabla \cdot(\mathbf{U})
$$

where $\alpha=\alpha_{1}$ is the volume fraction occupied by fluid $1,(1-\alpha)=\alpha_{2}$ the volume fraction occupied by fluid 2, $U$ is the velocity of the single effective fluid, $D$ is the molecular diffusion coefficient, $D_{T}$ is thermo-diffusion coefficient, $p$ is the pressure, $\psi$ is the compressibility and $\rho$ is the density of the fluid. The subscripts ' 2 ' and ' 1 ' correspond to the fluid 2 and fluid 1 respectively.

- Temperature equation (energy):

$$
\begin{aligned}
\frac{\partial}{\partial t}(\rho T)+\nabla \cdot(\rho U T)-\nabla \cdot\left(k_{e f f} \nabla T\right)-\nabla \cdot & \left(\frac{\left(\rho_{1} C_{v, 1}-\rho_{2} C_{v, 2}\right)}{C_{v, 12}}\left(D \nabla \alpha+D_{T} \nabla T\right) T\right) \\
& =-\frac{1}{C_{v, 12}}\left(\nabla \cdot(P U)+\frac{\partial}{\partial t}(\rho K)+\nabla \cdot(\rho U K)\right)
\end{aligned}
$$

where $T$ is the temperature, $K$ is the kinetic energy calculated by the solver as $K=$ $\left(|\mathbf{U}|^{2} / 2\right), C_{v, 1}$ and $C_{v, 2}$ are the specific heat capacities at constant volume for fluid 1 and fluid 2 respectively. The term $1 / C_{v, 12}$ is the inverse of heat capacity of the single effective fluid and is calculated as $\left(1 / C_{v, 12}=\alpha_{1} / C_{v, 1}+\alpha_{2} / C_{v, 2}\right)$

\section{Simulation cases and setup}

The simulated cases are presented in Table 2. The first set of cases, 1-4 corresponds to singlespecie/fluid configuration, where cryogenic $\mathrm{N}_{2}$ is injected into a chamber filled with warm $\mathrm{N}_{2}$ at 

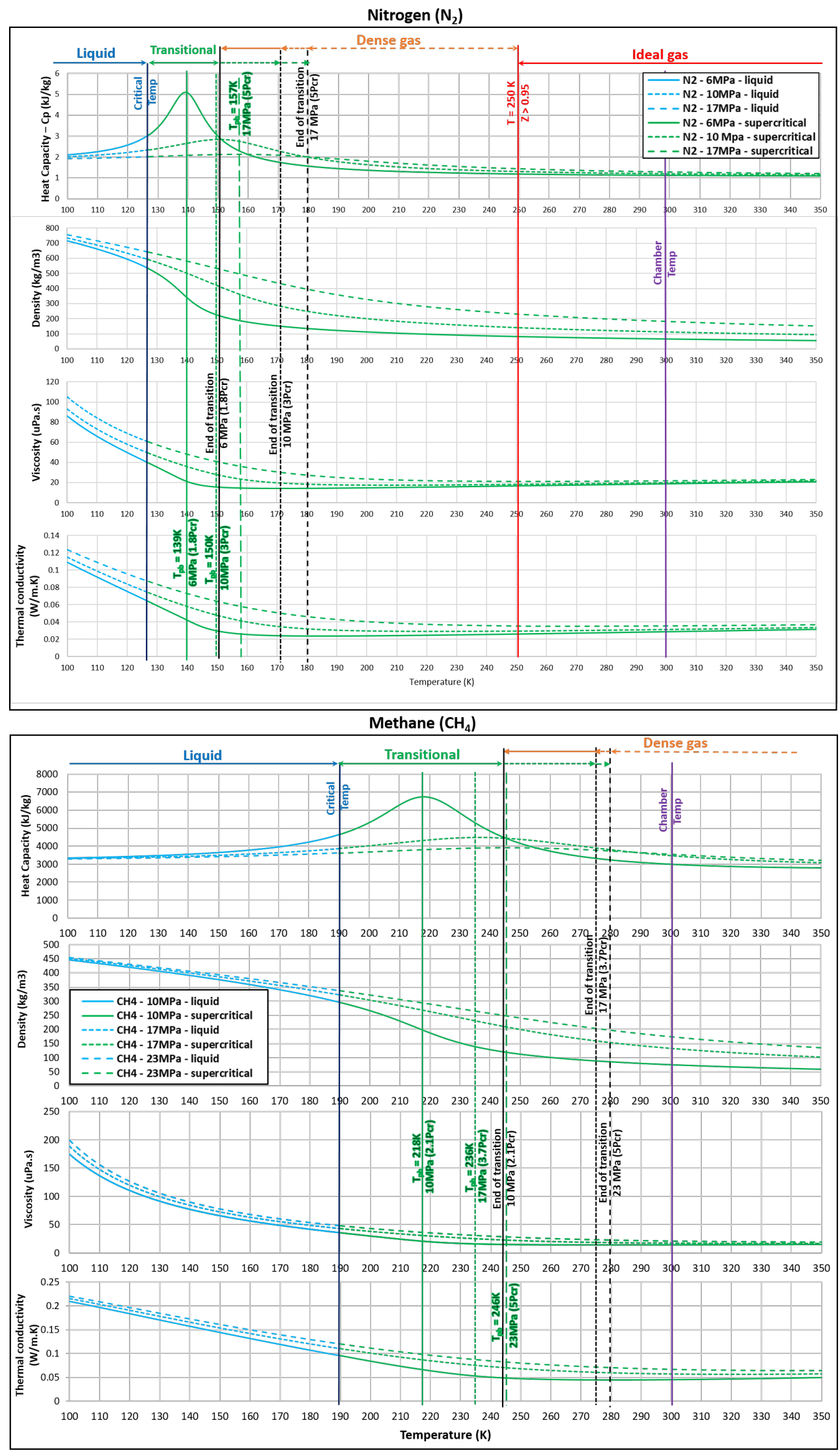

Figure 1. Classification of phases and pseudo-phases based on the isobaric thermo-physical variation against temperature for $\mathrm{N}_{2}$ (top) amd $\mathrm{CH}_{4}$ (bottom). The phase boundaries of the classification are presented for $N_{2}$ at pressures of $6 \mathrm{MPa}\left(1.8 P_{c r}\right), 10 \mathrm{MPa}\left(3 P_{c r}\right)$ and $17 \mathrm{MPa}\left(5 P_{c r}\right)$ and at pressures of $10 \mathrm{MPa}\left(2.1 P_{c r}\right), 17 \mathrm{MPa}$ $\left(3.7 P_{c r}\right)$ and $23 \mathrm{MPa}\left(5 P_{c r}\right)$ for $\mathrm{CH}_{4}$. The pseudoboiling point of the cryogenic fluids for respective supercritical pressures is also marked in vertical green lines. 


\begin{tabular}{|c|c|c|c|c|c|}
\hline \multirow{2}{*}{$\begin{array}{c}\text { Case } \\
\text { No }\end{array}$} & \multicolumn{2}{|c|}{ Injection } & \multicolumn{3}{|c|}{ Chamber } \\
\hline & Fluid & $\begin{array}{c}\text { Temp } \\
\text { (K) }\end{array}$ & Fluid & $\begin{array}{c}\text { Pressure } \\
\text { (MPa) }\end{array}$ & $\begin{array}{c}\text { Temp } \\
\text { (K) }\end{array}$ \\
\hline 1 & $\mathrm{~N}_{2}$ & $135\left(1.1 T_{c r}\right)$ & $\mathrm{N}_{2}$ & $6\left(1.8 P_{c r}\right)$ & $300\left(2.4 T_{c r}\right)$ \\
\hline 2 & $\mathrm{~N}_{2}$ & $135\left(1.1 T_{c r}\right)$ & $\mathrm{N}_{2}$ & $17\left(5 P_{c r}\right)$ & $300\left(2.4 T_{c r}\right)$ \\
\hline 3 & $\mathrm{~N}_{2}$ & $120\left(0.95 T_{c r}\right)$ & $\mathrm{N}_{2}$ & $6\left(1.8 P_{c r}\right)$ & $300\left(2.4 T_{c r}\right)$ \\
\hline 4 & $\mathrm{~N}_{2}$ & $120\left(0.95 T_{c r}\right)$ & $\mathrm{N}_{2}$ & $\left.17\left(5 P_{c r}\right)\right)$ & $300\left(2.4 T_{c r}\right)$ \\
\hline 5 & $\mathrm{CH}_{4}$ & $210\left(1.1 T_{c r}\right)$ & $\mathrm{N}_{2}$ & $10\left(2.1 P_{c r}\right)$ & $300\left(1.6 T_{c r}\right.$ \\
\hline 6 & $\mathrm{CH}_{4}$ & $210\left(1.1 T_{c r}\right)$ & $\mathrm{N}_{2}$ & $23\left(5 P_{c r}\right)$ & $300\left(1.6 T_{c r}\right)$ \\
\hline 7 & $\mathrm{CH}_{4}$ & $135\left(0.71 T_{c r}\right)$ & $\mathrm{N}_{2}$ & $10\left(2.1 P_{c r}\right)$ & $300\left(1.6 T_{c r}\right)$ \\
\hline 8 & $\mathrm{CH}_{4}$ & $135\left(0.71 T_{c r}\right)$ & $\mathrm{N}_{2}$ & $23\left(5 P_{c r}\right)$ & $300\left(1.6 T_{c r}\right)$ \\
\hline
\end{tabular}

Table 2. Table of cases of $\mathrm{N}_{2}$ at $135 \mathrm{~K}$ injected through a $2.2 \mathrm{~mm}$ diameter orifice into a chamber filled with gaseous $\mathrm{N}_{2}$ at $300 \mathrm{~K}$ for simulating various supercritical pressure conditions

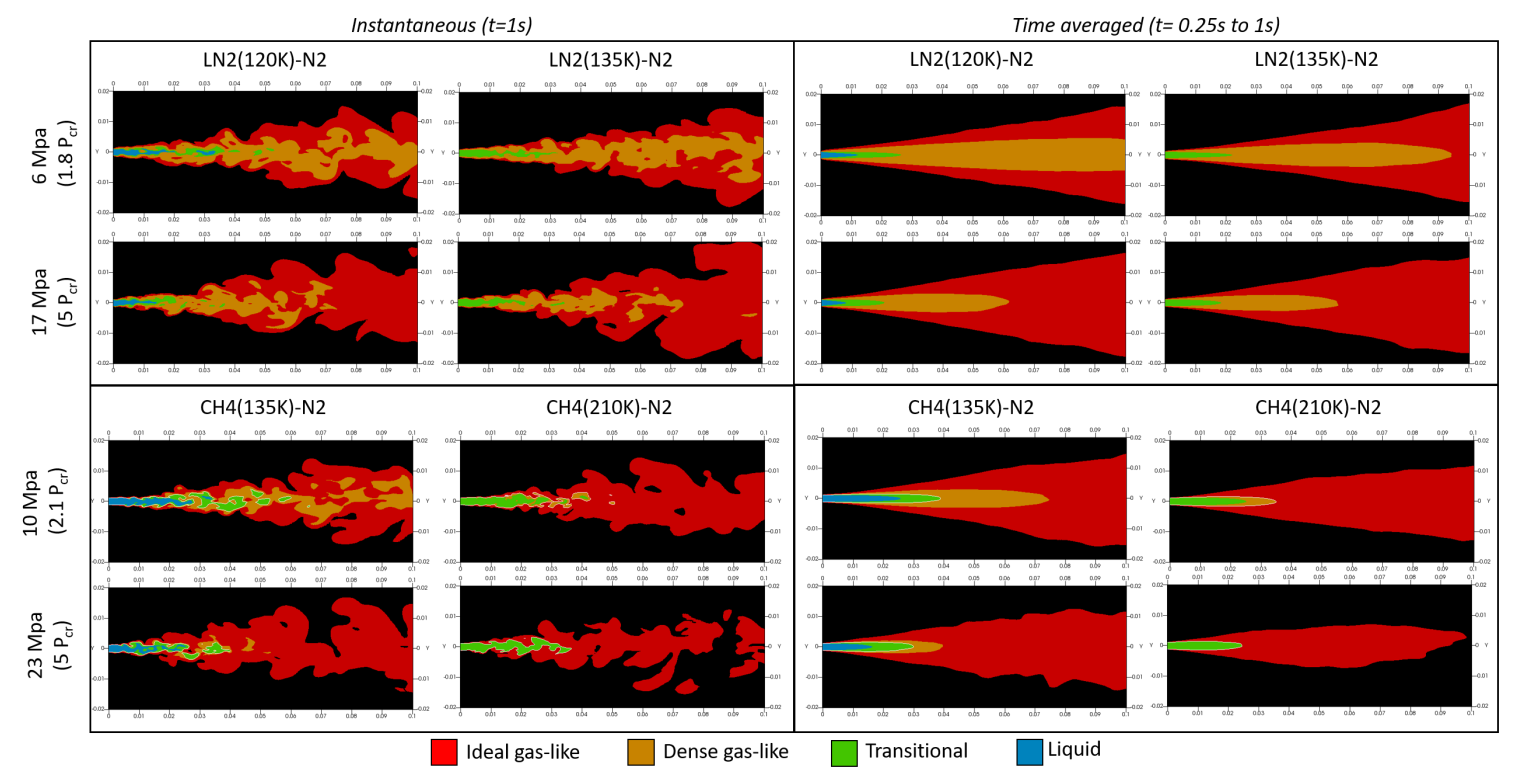

Figure 2. Distribution of phases in a developed supercritical cryogenic nitrogen jet (top) and methane jet (bottom) injected into warm nitrogen: instantaneous (left) time averaged (right)

$300 \mathrm{~K}$, as liquid (120K) and as supercritical fluid (135K) for three pressures. Second set of cases, 5-8 correspond to binary-specie/fluid configuration of $\mathrm{CH}_{4}$ injection into $\mathrm{N}_{2}$. Methane is injected at $135 \mathrm{~K}$ (as liquid) and $210 \mathrm{~K}$ (as supercritical fluid) into a chamber of $\mathrm{N}_{2}$ at $300 \mathrm{~K}$ for three chamber pressures. The injection velocity is $10 \mathrm{~m} / \mathrm{s}$ for all the cases and the pressures have been chosen to be representative of real cryogenic applications that these fluids are used. The chamber is constructed in the computational domain as 3D axi-symmetric cylindrical mesh where the fluid is injected through a inlet. The mesh resolution increases towards the axis as well as towards the inlet, while also ensuring sufficient resolution along the jet boundary. The mesh discretisation can be found in our previous publication[2], as we use the same mesh here. The instantaneous results are captured at $t=1 \mathrm{~s}$ and time averaged results are averaged in the duration $0.25 \mathrm{~s}$ to $1 \mathrm{~s}$ from injection. The real fluid thermophysical properties of $\mathrm{N}_{2}$ and $\mathrm{CH}_{4}$ for numerical simulations are estimated through polynomials, which are fit to isobaric NIST data. These polynomials of density, heat capacity, thermal conductivity and viscosity are used instead of an EoS (refer our earlier publication[2] for detailed explanation about this model).

\section{Results and Discussion}

The distribution of phases in cryogenic $\mathrm{N}_{2}$ and cryogenic $\mathrm{CH}_{4}$ jets into warmer $\mathrm{N}_{2}$ environment are presented in Fig. 2. The figure colouring is based on the classification of the phases of $\mathrm{N}_{2}$ and $\mathrm{CH}_{4}$ presented earlier in Fig. 1. In the $\mathrm{CH}_{4}$ into $\mathrm{N}_{2}$ case where binary fluid mixing 
occurs, the phase distribution (Fig. 2) and enthalpy distribution (3) is presented in such a way that up to $\alpha=0.5$ the fluid classification is considered based on $\mathrm{CH}_{4}$ and beyond $\alpha=0.5$ is considered based on $\mathrm{N}_{2}$. Supercritical phases: The distribution of supercritical phases (Fig. 2) provides insight into continuous phase transitions (at supercritical pressures) of cryogenic fluid while also being influenced by jet dynamics. $\mathbf{L N}_{2}-\mathbf{N}_{2}$ : At $6 \mathrm{Mpa}\left(1.8 P_{c r}\right)$, the instantaneous and time averaged pictures show that injection as a liquid $\left(\mathrm{T}_{i n j}=120 \mathrm{~K}\right)$ results in the transitional and dense gas-like phase regions penetrating deeper and occupying a larger region, when compared to the supercritical injection $\left(\mathrm{T}_{i n j}=135 \mathrm{~K}\right)$. This is because, at low supercritical pressures the drastic spike in heat capacity at $T_{p b}$, requires more thermal energy for the fluid to heat up and overcome the $T_{p b}$ in order to transition further. But at high supercritical pressures the pseudoboiling effect on the enthalpy and thermophysical properties is minimal as the variation is mostly linear beyond $3 P_{c r}$. As a result at $17 \mathrm{MPa}\left(5 \mathrm{P}_{c r}\right)$ even if the fluid is injected in a liquid state $\left(\mathrm{T}_{i n j}=120 \mathrm{~K}\right)$ the extent of the transitional and dense-gas like phases is comparable to supercritical injection $\left(\mathrm{T}_{i n j}=135 \mathrm{~K}\right.$ ), with only minor differences. $\mathbf{C H}_{4}-\mathbf{N}_{2}$ : At supercritical pressures when injected as liquid, $\mathrm{CH}_{4}$ heats up and transitions before mixing with chamber $\mathrm{N}_{2}$. The injected liquid $\mathrm{CH}_{4}$ cools down the surrounding gas $\mathrm{N}_{2}$ bringing it to a dense gas-like state. Whereas, for supercritical injection the $\mathrm{CH}_{4}$ does not cool the surrounding $\mathrm{N}_{2}$ to such an extent. The higher specific enthalpy of $\mathrm{CH}_{4}$ compared to $\mathrm{N}_{2}$ and the large heat absorption potential associated with lower temperatures is the reason for the surrounding $\mathrm{N}_{2}$ cooling down during liquid $\mathrm{CH}_{4}$ injection. The pseudoboiling effect in slow phase transition and increased penetration of phases is evident here as well. Overall, the strong pseudoboiling effect at low supercritical pressures slows phase transition and enhances phase penetration, whereas high supercritical pressures result in rapid transition to more gaseous like states.

Enthalpy and heat absorption: To understand the cooling effect of the cryogenic jets, the distribution of the enthalpy difference between the fluid in the cryogenic jet and the fluid at thermal equilibrium with chamber is presented in Fig. 3, along with the mean temperatures developed in the chamber due to the continuous injection of cryogenic fluid for the duration of $0.25 \mathrm{~s}$ to $1 \mathrm{~s}$. $\mathbf{L N}_{2}-\mathbf{N}_{2}$ : In both pressure cases injection as a liquid, results in large regions of lower temperatures being developed in the chamber due to the lower enthalpy associated with the injection state of $\mathrm{LN}_{2}$. The significant difference in the chamber temperature profiles between liquid and supercritical injection at $1.8 P_{c r}$ is because of the pseudoboiling effect. At $1.8 P_{c r}$ the variation in thermophysical properties (including enthalpy) is steep, whereas at high supercritical pressures $\left(>3 P_{c r}\right)$ variation in thermophysical properties becomes linear even around the $T_{p b}$. Thus, the enthalpy difference between $120 \mathrm{~K}$ and $135 \mathrm{~K}$ nitrogen is higher at $1.8 P_{c r}$. Interestingly though, the boundary of the temperature of $290 \mathrm{~K}$ is unchanged regardless of liquid or supercritical injection. $\mathbf{C H}_{4}-\mathbf{N}_{2}$ : Injection as liquid at 2.1 $P_{c r}$ results in increased extent of cool temperature profiles as opposed to injection as supercritical fluid. This is expected due to the added pseudoboiling effect at low supercritical pressures. Unlike the single fluid case $\left(\mathrm{LN}_{2}-\mathrm{N}_{2}\right)$, the boundaries of $290 \mathrm{~K}$ mean chamber temperature are not the same for liquid and supercritical injection at high supercritical pressures. Overall, in both the single and two fluid cases, the combination of low supercritical pressure environment (to allow for a strong pseudoboiling effect) and low temperature injection (below $T_{c r}$ ) to transition across the entire pseudoboiling point, results in enhanced heat absorption.

Mixing dynamics: To reveal the mixing dynamics and its relation with the pseudoboiling effect transition Fig 4 presents the isobaric heat capacity distribution along with the interface between the fluids/mixing location $(\alpha=0.5)$. $\mathbf{L N}_{2}-\mathbf{N}_{2}$ : The injected $\mathbf{N}_{2}$ in both liquid and supercritical cases overcomes the $T_{p b}$ before mixing with the chamber $\mathrm{N}_{2}$, which is evident by the $\max \left(C_{p}\right)$ values being present well within the mixing interface. $\mathbf{C H}_{4}-\mathbf{N}_{2}$ : The increased penetration of the interface reveals the suppressed binary mixing between $\mathrm{CH}_{4}$ and $\mathrm{N}_{2}$ at lower supercritical pressures and even more for liquid injection, similar to single fluid case. Unlike $L_{2} N_{2}-N_{2}$ injection, the injected liquid (135K) $\mathrm{CH}_{4}$ starts mixing with the chamber $\mathrm{N}_{2}$ before reaching $T_{p b}$ which 


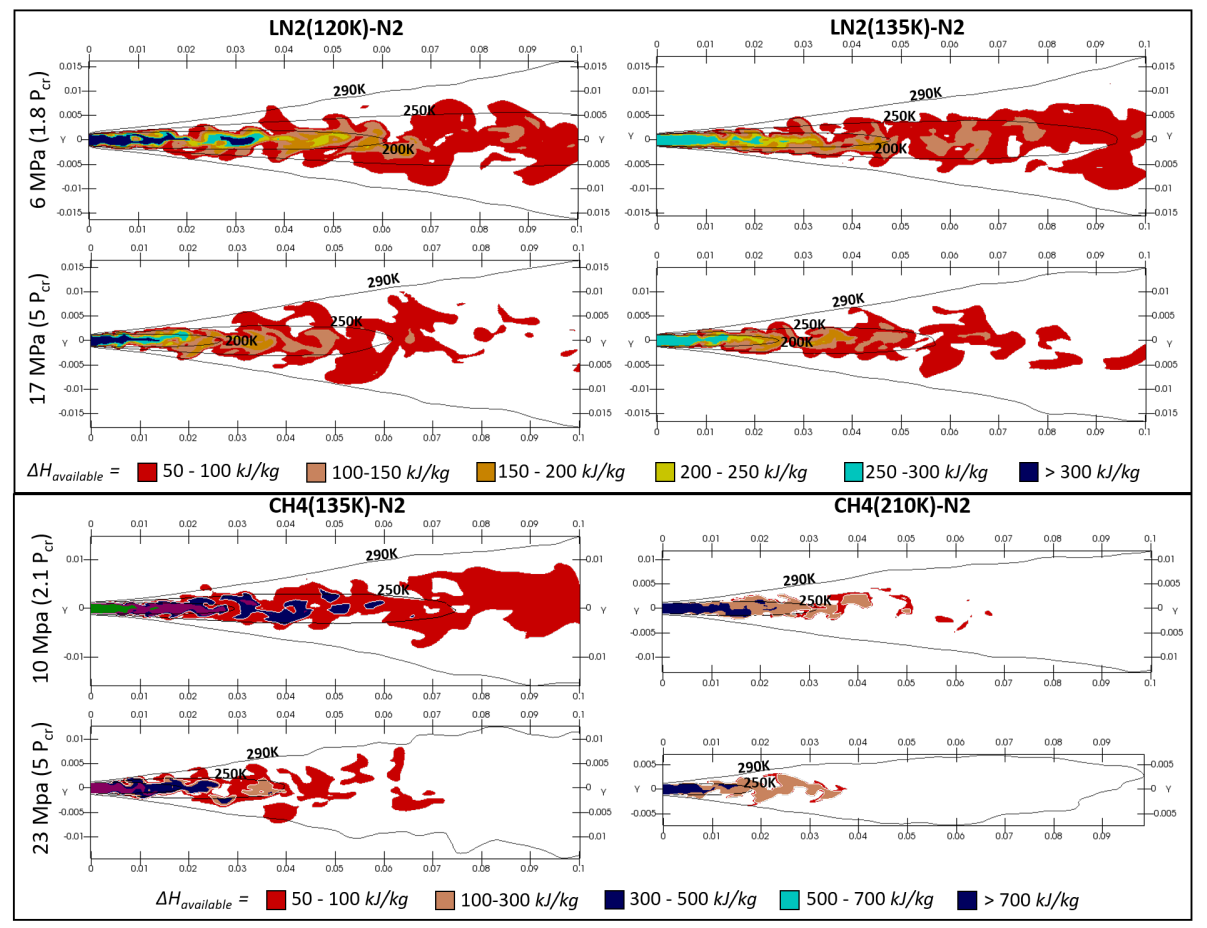

Figure 3. Available enthalpy difference (instantaneous) between the injected fluid and chamber fluid: LN2 into N2 (top) and $\mathrm{CH} 4$ into N2 (bottom). The mean temperature profile developed in the chamber due to the continuous injection of the respective cryogenic fluid is presented as black lines.

can be identified by the $\max C_{p}$ values coinciding with the interface. Furthermore, the $\max C_{p}$ values of supercritical injection are higher than the ones of liquid injection, which again indicates that $\mathrm{CH}_{4}$ starts mixing with chamber $\mathrm{N}_{2}$ before attaining the max $C_{p}$ at $T_{p b}$ for liquid injection. This is because $\mathrm{T}_{i n j}=135 \mathrm{~K}$ is below the $T_{c r}=190.55 \mathrm{~K}$ of methane and hence away from the pseudoboiling transition and $T_{p b}$. Overall, when injected close to $T_{c r}$ or $T_{p b}$ the pseudoboiling transition primarily determines the jet characteristics followed by mixing dynamics. So when enhanced mixing is required, high supercritical pressures and injection temperatures well above $T_{p b}$ are advantageous.

\section{Conclusions}

From the simulation results of both the single fluid $\left(\mathrm{LN}_{2}\right.$ into $\left.\mathrm{N}_{2}\right)$ and binary fluid jet $\left(\mathrm{CH}_{4}\right.$ into $\mathrm{N}_{2}$ ), the following conclusions are drawn.

- The additional energy required to overcome the pseudoboiling point at lower supercritical pressures is reflected in the delayed phase transition and increased heat absorption potential of cryogenic jets. The fluid phases penetrates deeper into the chamber as well, when injected as liquid at lower supercritical pressures. Thus injecting the cryogenic fluid at appropriate thermodynamic state to leverage the pseudoboiling effect makes them attractive as a coolant.

- As the pseudoboiling effect vanishes at high supercritical pressures, the increased penetration and heat absorption advantage of liquid injection also diminish proportionately, and the fluid in the jet transitions more rapidly to reach the temperature of the environment. As the pseudoboiling effect delays phase transition and suppresses mixing, if enhanced mixing is required, injection at high temperature (well above $T_{p b}$ ) and high supercritical pressure environment should be employed. 


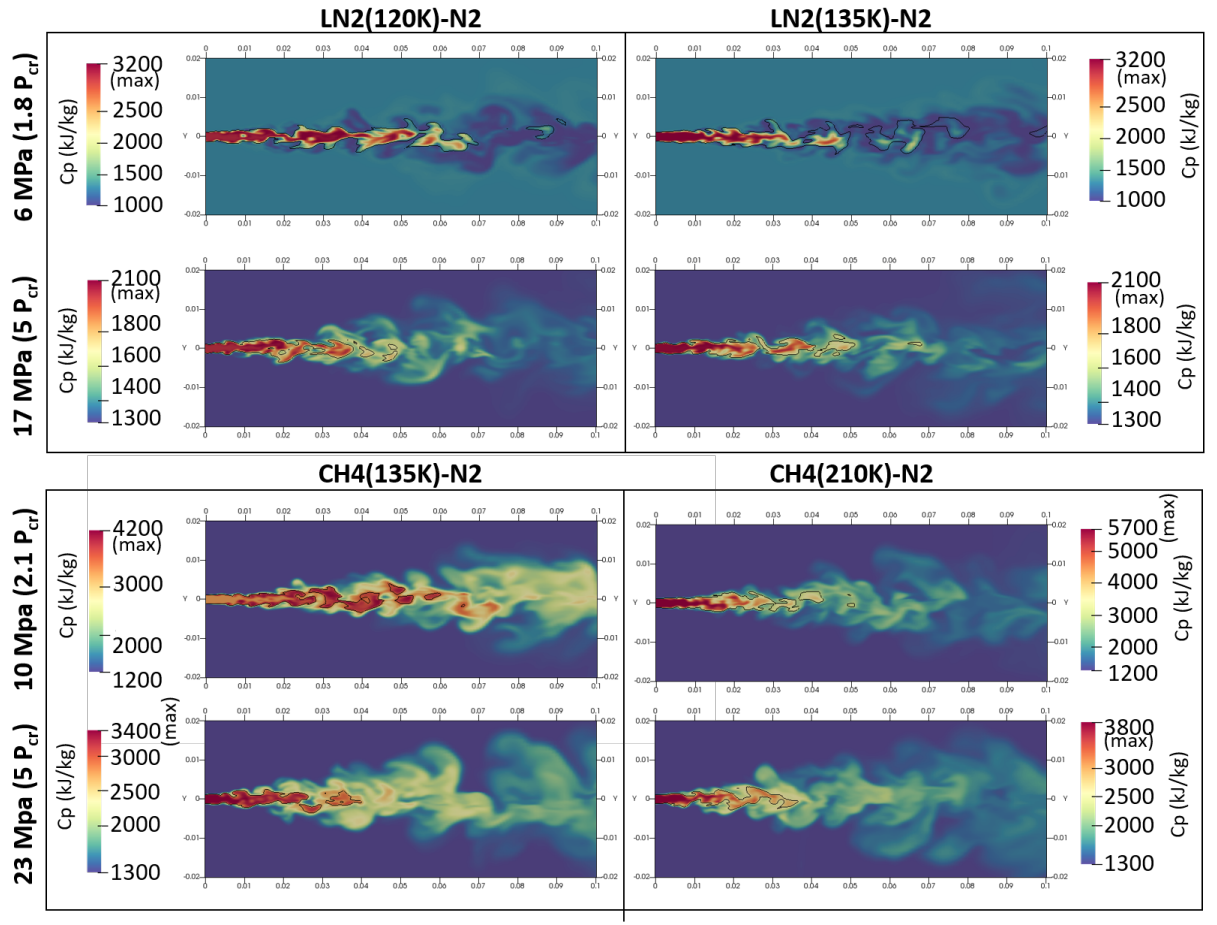

Figure 4. Heat capacity distribution with volume fraction ' $\alpha$ ' $=0.5$ located by black isoline.

- By changing the cryogenic fluid storage conditions (pressure and temperature) which links to the injection properties and thermodynamic state of the fluid, the penetration, heat absorption and mixing of supercritical cryogenic jets can be controlled and tuned to specific application requirements.

\section{Acknowledgements}

The authors would like to acknowledge funding by the UK Engineering and Physical Science Research Council support through the grant EP/S001824/1

\section{References}

[1] Jaya Vignesh, M., Harvey, S., Atkins, A., Atkins, P., De Sercey, G., Heikal, M., Morgan, R., and Vogiatzaki, K., in press 2019. "Use of cryogenic fluids for zero toxic emission hybrid engines". In Internal Combustion Engines and Powertrain Systems for Future Transport, IMEChE.

[2] Jaya Vignesh, M., Tretola, G., Morgan, R., Sercey, G. d., Atkins, A., and Vogiatzaki, K., 2020. "Understanding sub and supercritical cryogenic fluid dynamics in conditions relevant to novel ultra low emission engines". Energies, 13(12), p. 3038.

[3] Gerber, V., Baab, S., Förster, F. J., Mandler, H., Weigand, B., and Lamanna, G., 2020. "Fluid injection with supercritical reservoir conditions: overview on morphology and mixing.". The Journal of Supercritical Fluids, p. 105097.

[4] Föll, F., Gerber, V., Munz, C.-D., Weigand, B., and Lamanna, G., 2021. On the Consideration of Diffusive Fluxes Within High-Pressure Injections. Springer International Publishing, Cham, pp. 195-208.

[5] Banuti, D., Raju, M., and Ihme, M., 2020. "Between supercritical liquids and gases - reconciling dynamic and thermodynamic state transitions". The Journal of Supercritical Fluids, 165, p. 104895.

[6] Banuti, D. T., and Hannemann, K., 2016. "The absence of a dense potential core in supercritical injection: A thermal break-up mechanism”. Physics of Fluids, 28(3), p. 035103. 